\title{
LA EDUCACIÓN A DISTANCIA COMO MECANISMO DE INCLUSIÓN SOCIAL EN LAS UNIVERSIDADES DEL ECUADOR
}

\author{
DISTANCE EDUCATION AS A MECHANISM OF SOCIAL INCLUSION IN THE \\ UNIVERSITIES OF ECUADOR
}

\section{MARÍA SOLEDAD REA FAJARDO', JOSÉ ALBERTO MEDINA CRESPO²}

1 Universidad Católica de Santiago de Guayaquil.soledadreaf@gmail.com

2 Universidad Católica de Santiago de Guayaquil jose.medina@cu.ucsg.edu.ec

RESUMEN

La inclusión social en la educación superior es un tema que se encuentra en las políticas educacionales de algunos países latinoamericanos, entre ellos Ecuador. Las Instituciones de Educación Superior juegan un rol protagónico para el fortalecimiento del talento humano profesional que se necesita para el desarrollo de un país. Con la llegada de las Tecnologías de la Información y la Comunicación, la educación a distancia tomó más auge. Desde el punto de vista social, esta modalidad tiene la finalidad de dar acceso a aquellas personas que por diferentes limitantes no podrían asistir físicamente al aula de clases. La tecnología permite complementar lo presencial por lo virtual, propiciando que el espacio y tiempo se adapten a las necesidades de docentes y estudiantes. Además, de permitir que el estudiante se vuelva el protagonista de su aprendizaje y constructor del conocimiento. Los entornos virtuales de enseñanza - aprendizaje remplazan a los edificios. En fin, aparecen escenarios y herramientas digitales que potencian el aprendizaje colaborativo y el conocimiento abierto, aunque también generan controversias. A pesar de que hay muchos factores tecnológicos que facilitan la educación a distancia, es importante que se determinen las razones por las que hay personas que aún se sienten excluidas de las instituciones de educación superior. Por otro lado, las universidades ecuatorianas tienen el reto de articular a su gestión las nuevas políticas educacionales y buscar mecanismos para retroalimentar su pertinencia social.

PALABRAS CLAVE: inclusión social, entornos virtuales de enseñanzaaprendizaje, conocimiento abierto, educación superior a distancia.
ABSTRACT

Social inclusion in higher education is a topic that is in education policies of some Latin American countries, including Ecuador. Institutions of Higher Education (IHE) play a leading role in strengthening the professional human talent that is needed for a country's development. With the advent of information and communications technology (ICT), distance education took more booming. From the social point of view, this method is intended to provide access to those who for various constraints could not physically attend the classroom. Technology allows to complement the virtual classroom so, promoting that space and time to adapt the needs of teachers and students. In addition, allowing the students to be the protagonist of their learning and knowledge becoming a builder of it. Virtual teaching learning (VLE) are replacing buildings. Anyway, scenarios and digital tools enhance collaborative learning and open knowledge, but also generates controversies. Although there are many technological factors that facilitate distance education, it is important to determine the reasons why there are people who still feel excluded from the IES. Meanwhile, Ecuadorian universities are challenged to articulate new educational management policies and seek mechanisms for social relevance feedback.

KEYWORDS: social inclusion, virtual teaching-learning, open knowledge, distance higher education. 
INTRODUCCIÓN

La Unión Europea define la inclusión social como un proceso que asegura que aquellos en riesgo de pobreza y exclusión social, tengan las oportunidades y recursos necesarios para participar completamente en la vida económica, social y cultural, disfrutando un nivel de vida y bienestar que se considere normal en la sociedad en la que ellos viven. Se considera que los estudios a distancia permiten una inclusión en la educación superior para las personas que tienen limitantes de tiempo y/o espacio, discapacidades físicas o cualquier otro factor que impida su asistencia a un aula de clases en un horario determinado. Por eso, desde el punto de vista social, tiene la finalidad de dar acceso a la educación a aquellas personas que no lo pueden hacer de forma presencial. A pesar de que las políticas educacionales para la inclusión en la educación superior en algunos países de Latinoamérica han mostrado resultados positivos, también han generado nuevas dimensiones de exclusión. Esto se debe a que no se ha asumido el carácter multidimensional de ésta. Las Instituciones de Educación Superior (IES) son elementos clave en las estrategias de desarrollo (Núñez, 2010).

En el artículo 43 del Reglamento del Régimen Académico del Ecuador, se define a la modalidad a distancia como aquella en la cual el componente de docencia, el de prácticas de los aprendizajes y la parte autónoma están medidos por el uso de tecnologías y entornos virtuales y por la articulación de recursos educativos. Su característica principal es que conjuga algunos elementos como son los docentes y los estudiantes; que utilizan espacios tecnológicos para intercambiar información de forma asincrónica. Es decir, los salones físicos se sustituyen por las aulas virtuales y el tiempo de estudio es manejado por los estudiantes de acuerdo a su propia planificación.

En Ecuador, la educación a distancia nació en el año 1970, a través de la radio como medio difusor. En los programas del Instituto $\mathrm{Ra}-$ diofónico Fe y Alegría se transmitían conocimientos cotidianos u ocupacionales y algunas clases de educación básica hasta tercer año. En 1976, la Universidad Técnica Particular de Loja inicia la modalidad a distancia para personas que no podían asistir por sus horarios de trabajo (Torres, 2002). Actualmente, existen otras universidades que se han sumado a esta oferta educativa como la Universidad Católica de Santiago de Guayaquil, la Universidad de Especialidades Espíritu Santo, la Universidad Internacional del Ecuador, la Universidad San Francisco de Quito, la Universidad de Cuenca, y la Escuela Politécnica del Ejército, entre otras.

En este artículo se busca caracterizar el papel de la educación a distancia como mecanismo de inclusión social en las universidades del Ecuador. Para lograrlo se revisarán los presupuestos teóricos de esta modalidad y el aprendizaje colaborativo desde una mirada nacional. Además, se destacará la importancia de los recursos educativos y de las prácticas educativas abiertas. Por último, se revisará lo que está haciendo el gobierno y las diferentes articulaciones que son necesarias para dar un marco referencial para el desarrollo de la modalidad a distancia.

\section{PANORAMA NACIONAL}

En Ecuador existe el Consejo de Educación Superior (CES), que es la institución que da las directrices para las universidades. A pesar de que el gobierno se preocupa por dar oportunidades para los estudios de educación superior, algunas personas presentan diferentes razones para no asistir a un establecimiento educativo. El Instituto Nacional de Estadísticas y Censos (INEC), la Comisión de Transición hacia el Consejo de la Igualdad de Género y ONU Mujeres Ecuador (2013) recopilaron algunas de ellas en su libro "Mujeres y Hombres del Ecuador en Cifras III".

En la Tabla 1, se muestra que las mujeres no pueden asistir a un centro educativo por los siguientes factores: falta de recursos económicos $(30.20 \%)$, trabajo $(20.70 \%)$, quehaceres del hogar $(17.70 \%)$. Por otro lado, en los hombres incide en una mayor proporción el trabajo $(40.00 \%)$ y la falta de recursos económicos (27.70\%). Además, se presentan en ambos casos otras razones como la discapacidad y la edad; que a veces por vergüenza se sentirían en desventaja con sus compañeros en un salón de clases. Por lo tanto, se puede destacar que la educación presencial, aún no logra superar muchas de estas razones. En cambio, la modalidad a distancia ofrece ventajas para superar algunas de ellas. El factor económico, es un tema común en ambos géneros y por esto sería muy importante que existan programas gratuitos o a un bajo costo. 
TABLA 1. RAZONES DE NO ASISTENCIA A UN ESTABLECIMIENTO EDUCATIVO

\begin{tabular}{|c|c|c|c|c|c|}
\hline \multirow{2}{*}{$\begin{array}{l}\text { RAZONES DE NO } \\
\text { ASISTENCIA }\end{array}$} & \multicolumn{2}{|c|}{ MUJER } & \multicolumn{2}{|c|}{ HOMBRE } & \multirow{2}{*}{$\begin{array}{r}\% \text { DE } \\
\text { MUJERES CON } \\
\text { RELACIONES A } \\
\text { LAS RAZONES } \\
\text { DE NO } \\
\text { ASISTENCIA }\end{array}$} \\
\hline & $\mathbf{N}$ & $\%$ & $\mathbf{N}$ & $\%$ & \\
\hline Edad & 2.890 & 0,5 & 2.673 & 0,4 & 51,9 \\
\hline $\begin{array}{l}\text { Terminó sus } \\
\text { estudios }\end{array}$ & 25.249 & 4,4 & 16.741 & 2,4 & 60,1 \\
\hline $\begin{array}{l}\text { Falta recursos } \\
\text { económicos }\end{array}$ & 172.965 & 30,2 & 191.393 & 27,7 & 47,5 \\
\hline Fracaso escolar & 10.221 & 1,8 & 10.607 & 1,5 & 49,1 \\
\hline Trabajo & 118.486 & 20,7 & 304.187 & 44,0 & 28,0 \\
\hline Temor maestros & 779 & 0,1 & 709 & 0,1 & 52,3 \\
\hline $\begin{array}{l}\text { Enfermedad o } \\
\text { discapacidad }\end{array}$ & 19.393 & 3,4 & 29.699 & 4,3 & 39,7 \\
\hline $\begin{array}{l}\text { Quehaceres del } \\
\text { hogar }\end{array}$ & 101.332 & 17,7 & 3.406 & 0,5 & 36,5 \\
\hline $\begin{array}{l}\text { Familia no } \\
\text { permite }\end{array}$ & 18.316 & 3,2 & 919 & 0,1 & 96,7 \\
\hline $\begin{array}{l}\text { No hay } \\
\text { establecimientos } \\
\text { educativos }\end{array}$ & 3.373 & 0,6 & 3.068 & 0,4 & 95,2 \\
\hline $\begin{array}{l}\text { No está } \\
\text { interesado }\end{array}$ & 42.534 & 7,4 & 75.375 & 10,9 & 52,4 \\
\hline Por embarazo & 14.051 & 2,5 & 0 & 0,0 & 36,1 \\
\hline Por falta de cupo & 15.627 & 2,7 & 20.385 & 3,0 & 100,0 \\
\hline Por otra razón & 26.799 & 4,7 & 31.555 & 4,6 & 43,4 \\
\hline Total & 572.015 & 100,0 & 690.718 & 100,0 & 45,9 \\
\hline
\end{tabular}

Fuente: Instituto Nacional de Estadísticas y Censos, Comisión de Transición hacia el Consejo de la lgualdad de Género y ONU Mujeres Ecuador (2013).

Las políticas para la inclusión en las universidades latinoamericanas han mostrado avances positivos, pero también han generado nuevas dimensiones de exclusión, al no asumir su carácter multidimensional. No se trata sólo de pobreza en un nivel económico sino cultural, social, educativo, etc. Tener acceso a la vivienda, empleo, una nutrición adecuada y otros factores necesarios como la brecha digital y el acceso a la información también puede afectar el acceso a la educación superior. Por lo tanto, las personas se preocuparán más por superar esas condiciones, que por estudiar y podrían seguir excluidos de la educación superior.

De manera general, es importante resaltar el perfil de los alumnos de la modalidad a distancia de las universidades ecuatorianas donde sobresalen amas de casa, personas de comunidades que no tienen un fácil acceso a la educación universitaria, trabajadores que sus horarios laborales les impiden asistir a un lugar específico en un tiempo determinado y personas privadas de libertad. La inclusión tiene varias aristas como el acceso, la participación y los logros de los estudiantes, dando prioridad a aquellos que tengan más riesgo de ser excluidos o marginados (UNESCO, 2005). Por lo tanto, es prioritario potenciar la gestión del conocimiento para que los futuros profesionales mejoren la calidad de vida de sus familias e impactar positivamente en el desarrollo de la sociedad y la productividad del país.

Brennan, Edmunds, Houston, Jary, Lebeau, Osborne \& Richardson (2010) manifiestan que el gran reto de la formación en la educación superior es atender la gran diversidad de estudiantes y ser consciente de las diferencias de clase, etnicidad, edad, aspiraciones, educación y pluralidad de circunstancias vitales. Por eso, es importante contemplar lo más conveniente para el estudiante de la modalidad a distancia; además de atender las razones por las cuales la educación presencial no les favorece en la superación de sus limitantes.

Otro punto clave para la educación a distancia, es el uso de las TIC y con ello de los entornos virtuales de enseñanza-aprendizaje (EVEA). A partir del mismo se debe establecer un modelo de actuación pedagógica que marque las pautas de acción de toda la comunidad educativa. Este modelo se debe centrar en el estudiante, y tendrá el entorno de relación como medio, pero no como finalidad en sí misma (Duart y Sagrá, 2000). Las Instituciones de Educación Superior tienen la ardua tarea de incursionar en modelos educativos más explícitos y organizativos acorde a las exigencias de los estudiantes. Las TIC son relevantes para la educación a distancia (EaD), pero sus verdaderos protagonistas son los docentes y los estudiantes.

Por otro lado, el acceso a internet y a las computadoras en poblaciones rurales es muy limitado; en especial en las poblaciones más alejadas de las ciudades principales. Todavía hay personas que no tienen o no pueden manejar de manera adecuada una computadora, teléfono inteligente, tableta $\mathrm{u}$ otro dispositivo tecnológico para la comunicación. Para el Instituto Nacional de Estadísticas y Censo del Ecuador (2013), se considera que una persona es un analfabeto digital si cumple simultáneamente las siguientes características:

1. No tiene celular activado.

2. No ha utilizado una computadora en los últimos doce meses.

3. No ha utilizado internet en el último año. 
Porcentaje de personas analfabetas digitales por área

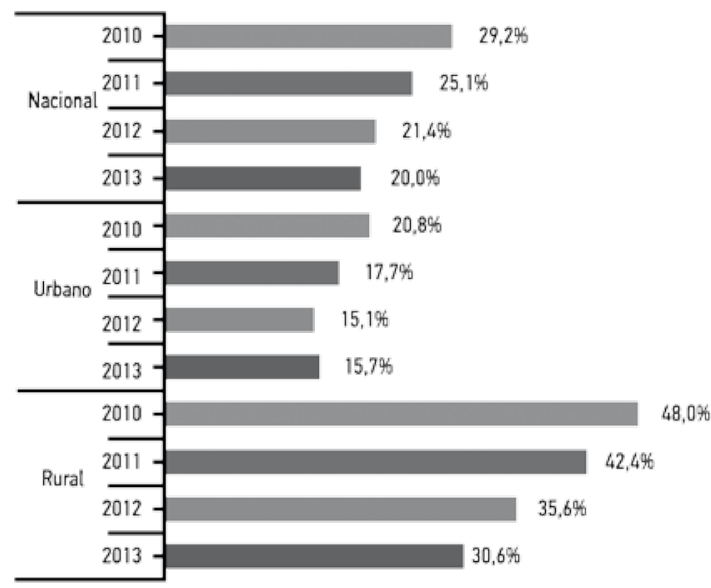

Figura 1. Porcentaje de personas analfabetas digitales por área en el Ecuador

En la Figura 1, tomada del Instituto Nacional de Estadísticas y Censos (2013), se puede apreciar que en el año 2013, en el área urbana sólo un $15.50 \%$ de la población es considerada analfabeta digital. En comparación, con el $30.60 \%$ de la población rural existe una gran diferencia. Esto contempla que el acceso a internet y a las computadoras es menor en este segundo sector. Por esto, para que la educación a distancia sea de alcance global a nivel país, el Ecuador debe propiciar políticas públicas a favor del acceso a internet y el uso de las computadoras.

El reto para el futuro será emplear todo el potencial de las nuevas tecnologías de acuerdo con estrategias instruccionales y educativas claras (Roll, 1995). Los modelos centrados en el estudiante le deben permitir ejercer la libertad de aprovechar al máximo el apoyo que se le ofrece, de planificar su progreso de aprendizaje universitario y de regular su propio ritmo de trabajo (Duart y Sangrá, 2000). En la educación a distancia, se debe procurar que el estudiante participe de manera activa en su educación y el docente propicie actividades colaborativas con el objetivo de que los estudiantes construyan su propio conocimiento. Además, la posibilidad de utilizar nuevos métodos en el proceso de enseñanza - aprendizaje, y aumentar la colaboración en las diferentes fases organizativas del proceso docente educativo (Castañeda, 2002). Por lo tanto, la colaboración potencia la comunicación entre docentes, estudiantes y docentes, y entre estudiantes.

El conocimiento es visto como un constructo social, y por tanto el proceso educativo es caracterizado por la interacción social en un entorno que facilita la interacción, la evaluación y la cooperación entre iguales (Hiltz y Turoff, 1993).
El conocimiento es un bien que al compartirse mantiene su característica creadora de generar más conocimiento, a diferencia de otros bienes que al compartirse se dividen. En la educación, el aprendizaje colaborativo invita a dar y recibir experiencias, información y conocimiento para lograr un aprendizaje significativo. Para que exista se deben propiciar momentos de interacción del sujeto que aprende y los demás actores (Vigotsky, 1997).

\section{EL SECTOR PÚBLICO: ARTICULACIÓN}

El gobierno ecuatoriano se encuentra impulsando políticas públicas a favor del acceso a las telecomunicaciones y la erradicación de la brecha digital. En esta vía, el Ministerio de Telecomunicaciones y de la Sociedad de la Información (2013) busca el despliegue masivo de banda ancha en todos los estratos sociales de forma inclusiva y solidaria. Además, destaca que esto influye de forma positiva en el cambio de la matriz productiva, la disminución de la pobreza y el desempleo. El manejo de herramientas tecnológicas abre una gama de oportunidades para acceder a la capacitación, adiestramiento y formación a distancia. Es por esto, que se deben incorporar puntos de acceso a internet gratuitos para estudiantes y mecanismos para superar la llamada brecha digital.

Se están dando grandes pasos en búsqueda de políticas educacionales a favor de la inclusión social en las universidades. Se ven avances significativos en la modalidad presencial, ya que actualmente se cuenta con una normativa técnica-legal a favor de algunos aspectos en pro de la inclusión de grupos vulnerables como: mujeres embarazadas y personas con capacidades especiales (PCE). En el artículo 47, numeral 7, de la Constitución de la República del Ecuador (2008) se menciona que el Estado garantizará a las personas con discapacidad una educación que desarrolle sus potencialidades y habilidades y que los establecimientos educativos cumplirán normas de accesibilidad. Por ejemplo, se han implementado parqueos y rampas para facilitar el acceso y la movilidad de estas personas. A pesar de que en el artículo 40 del Reglamento del Régimen Académico, expedido por el Consejo de Educación Superior (2015) se indica que en cada una de las modalidades se debe asegurar que las personas con capacidades diversas tendrán el derecho a recibir una educación que incluya recursos, medios y ambientes de aprendizaje apropiados para potenciar sus capacidades, aún muchos no pueden realizar 
sus estudios universitarios porque el desplazamiento desde sus hogares hasta los centros educativos presenta peligros o no pueden costear su movilización; además de sumar el miedo a las burlas que pueden sufrir de parte de algunos de sus compañeros. Factores como los expuestos anteriormente abren el camino para propiciar encuentros virtuales asincrónicos pero aún queda una interrogante: ¿La oferta educativa a distancia de las universidades públicas es suficiente para satisfacer la demanda?

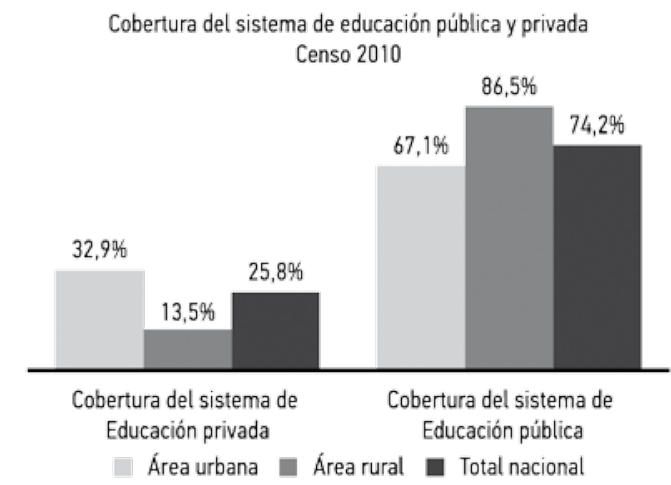

Figura 2. Cobertura del sistema de educación privado y público en el Ecuador

En la Figura 2, tomada del INEC (2010), se muestra que la mayor población estudiantil asiste a las instituciones públicas. En el área rural, esta representa un $86.50 \%$ y se debe a que la mayoría no cuenta con recursos suficientes para acceder a la educación privada. Aunque en el sector urbano, el acceso a la educación privada es mayor que en el rural, hay un $67.10 \%$ que asisten a instituciones públicas. Además, hay que considerar que las desigualdades tienen un origen socioeconómico y la pobreza está asociada a vivir en zonas rurales o pertenecer a pueblos originarios, lo cual sitúa a una buena parte de la población latinoamericana en una posición de vulnerabilidad (Blanco, 2006).

DISCUSIÓN

El avance en Ciencia, Tecnología e Innovación (CTI) en la educación superior en Latinoamérica es todavía bajo, pero se encuentra en crecimiento. Una muestra de ello es la categorización de las universidades ecuatorianas, donde se evalúa la calidad para garantizar una auténtica oferta educativa. Desde el 2012, el Consejo de Evaluación, Acreditación y Aseguramiento de la Calidad de la Educación Superior (CEAACES) emprendió la búsqueda de la excelencia y en su primera fase se cerraron 14 universidades que se encontraban en la categoría $\mathrm{E}$, la misma que indica pésima calidad con miras a la extinción. Por esto, si a veces algunos educadores se quejan de una mala calidad de la educación a distancia, también deberían reflexionar sobre esas 14 instituciones que tenían a sus docentes y alumnos de forma presencial. Por lo tanto, no se trata de modalidades de estudios, sino de modelos pedagógicos que funcionen e incentiven a que los alumnos desarrollen las competencias necesarias para gestionar el conocimiento a lo largo de su vida.

Es muy importante, que las universidades se relacionen con su entorno. Así lo manifestaron autores como Sábato y Botana (1986) donde se aprecia la relación entre universidad, sociedad y estado. En cambio, después de algunos años en el modelo de Etkowitz y Leydesdorff (1995) se remplaza a la sociedad por la empresa. Por lo tanto, los gobiernos deberían propiciar políticas educacionales para las universidades y estas deben ser pertinentes con la sociedad; además de permitir un intercambio dinámico entre los tres sectores. En la Figura 3, se propone una adaptación al triángulo presentado por Sábato en 1968, a través de un modelo dinámico de gestión universitaria donde el gobierno, las universidades y la sociedad se articulan en doble vía. Por ejemplo, cuando las necesidades de la sociedad cambian el gobierno interviene fijando el marco legal que sirva de base para que las universidades ajusten su oferta educativa y todos los componentes se retroalimentan constantemente.

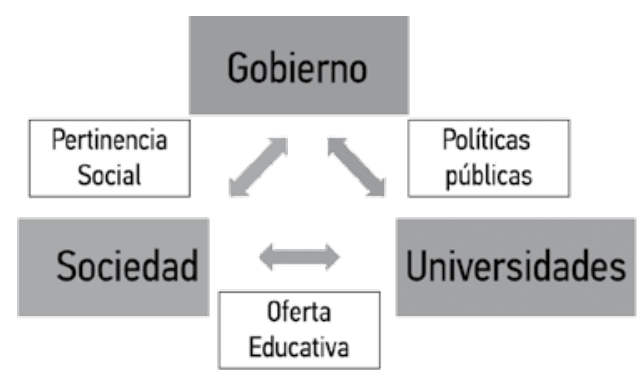

Figura 3. Modelo dinámico de gestión universitaria.

Este modelo se aplica a cualquier modalidad de estudios. Además, el gobierno, la sociedad y las universidades deben crear una sinergia para estar a la vanguardia de la oferta educativa nacional e internacional. El Consejo de Educación Superior (2015) presentó el reglamento para carreras y programas académicos en modalidades en línea, a distancia, semipresencial y en convergencia de medios. El mismo fue trabajado en conjunto con las universidades ecuatorianas que ofertan esta modalidad y es un gran avance para lograr esta articulación dinámica entre los actores. 


\section{CONCLUSIONES}

La educación superior a distancia tiene algunos retos que superar en el Ecuador. El Estado debe fomentar políticas educacionales a la vanguardia de las necesidades de la sociedad. Las universidades que ofertan esta modalidad tienen la tarea de generar modelos pedagógicos que propicien la gestión del conocimiento. El gobierno deberá trabajar en conjunto con las IES para disminuir las brechas socioeconómicas, a través del conocimiento abierto y gratuito para los sectores más vulnerables de la sociedad.

Por último, se destaca que las TIC por sí solas no cambiarán la educación. Sin embargo, generan una gran revolución que con una correcta integración de sus elementos ayudarán a las universidades a formar los profesionales acordes a las exigencias del mundo actual. La educación a distancia debe estar a la vanguardia, ya que las tecnologías de información y comunicación avanzan a pasos de gigantes, al igual que sus nuevos retos. A mayor inversión en educación superior, el bienestar se refleja en la sociedad. La EaD es en definitiva una modalidad que representa la inclusión social. Por lo tanto, debe ser tomada en cuenta como una estrategia para aumentar el acceso a la educación superior en Ecuador.

\section{REFERENCIAS BIBLIOGRÁFICAS}

Blanco, R. (2006). La equidad y la inclusión social: Uno de los desafíos de la educación y la escuela de hoy. Madrid: REICE.

Brennan, J., Edmunds, R., Houston, M., Jary, D., Lebeau, Y., Osborne, M. and Richardson, J. (2010). Improving what is learned at university: An exploration of the social and organisational diversity of university education. London, UK: Routledge.

Castañeda, A. (2002). Las nuevas tecnologías de la información y telecomunicaciones como proceso cultural y las bases de su impacto en la actividad educativa. Conferencia Internacional Un acercamiento desde lo tecnológico. Universidad Técnica de Ambato, Ambato. $25 \mathrm{pp}$.

Consejo de Educación Superior. (2015). Reglamento del Régimen Académico. Recuperado de http:// www.ces.gob.ec/doc/Reglamentos_Expedidos_CES/ codificacin $\% 20$ del $\% 20$ reglamento $\% 20$ de $\% 20$ rgimen\%20acadmico.pdf

Constitución de la República del Ecuador. (2008). Recuperado de http://www.oas.org/juridico/pdfs/ mesicic4_ecu_const.pdf

DuartJ. y Sangrá A. (2000). Aprender en la virtualidad. Recuperado de http://www.terras.edu.ar/aula/ cursos/3/biblio/DUART_Joseph_y_SAGRA_Albert-
Formacion_Universitaria_por_medio_de_la_webun_modelo_integrador.pdf

Instituto Nacional de Estadísticas y Censos (2013). Encuesta Nacional de Empleo Desempleo y Subempleo - ENEMDU (2010 - 2013). Recuperado de: http:// www.ecuadorencifras.gob.ec/documentos/webinec/Estadisticas_Sociales/TIC/Resultados_principales_140515.Tic.pdf

Etzkowitz, H., y Leydesdorff, L. (1995). The Triple Helix University Industry Gobernment Relations: A laboratory for Knowledge based Economic Development. EASST Review, 14, 14-19.

Hiltz S y Turoff M. (1993). The virtual classroom: CMC for collaborative learning. Recuperado de: http:// penta.ufrgs.br/edu/telelab/12/dised2.htm

Instituto Nacional de Estadísticas y Censos, La Comisión de Transición hacia el Consejo de la Igualdad de Cénero y ONU. (2013). Mujeres Ecuador. Mujeres y Hombres del Ecuador en cifras III. Recuperado de http://www.igualdadgenero.gob.ec/ publicaciones/150-cifras-iii.html

Instituto Nacional de Estadísticas y Censos. (2013). Tecnología de la Información y Comunicaciones. Recuperado de http://www.ecuadorencifras.gob. ec/documentos/web-inec/Estadisticas_Sociales/ TIC/Resultados_principales_140515.Tic.pdf

Ministerio de Telecomunicaciones y de la Sociedad de la Información. (2013). La penetración de internet banda ancha aumentó en el Ecuador y se consolida como política pública. Recuperado de http://www. telecomunicaciones.gob.ec/la-penetracion-deinternet-banda-ancha-aumento-en-el-ecuador-yse-consolida-como-politica-publica/

Núñez, J. (2010). Las políticas de posgrado, sus fundamentos conceptuales y la larga batalla contra el subdesarrollo. En Mollis, M; Núñez, J y García Guadilla, C. (eds.), Políticas de posgrado y conocimiento público en América Latina y el Caribe (pp. 57-134). Buenos Aires: CLACSO.

Roll, R. (1995). Tendencias internacionales en el aprendizaje abierto y a distancia. Barcelona: Universidad Abierta de Cataluña.

Sábato, J., y Botana, N. (1968). La ciencia y la tecnología en el desarrollo futuro en América Latina. The World Order Models Conference. Bellagio, Italia.

Torres, J. (2002). Digital observatory for higher education in latin america and the caribbean. Recuperado de http://unesdoc.unesco.org/ images/0014/001404/140469s.pdf

UNESCO. (2005). Directrices sobre políticas deinclusión en la educación. Recuperado de http://unesdoc. unesco.org/images/0017/001778/177849s.pdf

Vigotsky L. (1997). Obras escogidas (Tomo 1, 2da ed.). Madrid: Aprendizaje Visor. 